\title{
On the Effect of Human Body Parts in Large Scale Human Behaviour Recognition
}

\author{
O. V. Ramana Murthy ${ }^{1}$, Ibrahim Radwan ${ }^{1}$, Abhinav Dhall $^{2}$ and Roland Goecke ${ }^{1,2}$ \\ ${ }^{1}$ Vision \& Sensing, HCC Lab, ESTeM, University of Canberra \\ ${ }^{2}$ IHCC, RSCS, CECS, Australian National University \\ Email: O.V.RamanaMurthy@ieee.org, ibrahim.radwan@canberra.edu.au, abhinav.dhall@anu.edu.au, roland.goecke@ieee.org
}

\begin{abstract}
Automatic analysis of human behaviour in large collections of videos is gaining interest, even more so with the advent of file sharing sites such as YouTube. Human behaviour analysis methods can be categorised into three classes based on the type of features. The three representations are local, region of interest and densely sampled based representations. Local feature representation, such as Spatio-Temporal Interest Points (STIP), are quite popular for modelling temporal aspects in human action recognition. Region of Interest (ROI) based feature representations try to capture and represent human body part regions. Densely sampled representations capture information at uniformly spaced intervals spread in space and temporal directions of the given video. In this paper, we investigate the effect of human body part (ROI) information in large scale action recognition. Further, we also investigate the effect of its fusion with Harris 3D points (local representation) information and densely sampled representations. All experiments use a Bagof-Words framework. We present our results on large class benchmark databases such as the UCF50 and HMDB51 datasets.
\end{abstract}

\section{INTRODUCTION}

Video data is an emerging popular source of media for information, education, entertainment etc. With the advent of the internet in the recent decades, users are uploading millions of videos on social networking and file sharing sites such as YouTube and Facebook. This has created challenges for efficient video mining. Video mining can be broadly divided into the following sub-tasks: interpretation of objects, actions, scenes, video summarisation and retargeting. This paper deals with videos containing human body motion with an objective of action recognition. There are several online and offline applications of human action recognition. Offline applications include event detection such as injury detection in rugby matches; efficient video retrieval such as spotting a person in news footage; and video indexing. Online applications include automatic surveillance in public places, shopping malls, hospitals / aged care; interactive applications such as video games, robotics and so on.

Feature representation is very important in any classification problem. On the basis of feature representation, we divide existing representations into three major categories: local, region of interest (ROI) and dense sampling. Local representations are obtained in a bottom-up fashion: SpatioTemporal Interest Points (STIP) are detected first and local patches are calculated around these points. Finally, the patches are accumulated over the entire video to yield the video's descriptor. A good example are the Harris 3D corners proposed by Laptev et al. [1] as an extension of the traditional
(2D) Harris corner points. However, the drawback of these representations is that the detected points are sometimes too few to sufficiently capture and model the human actions. For instance, in a basketball clip (UCF dataset) as shown in Fig. 1 (top row), very few or no points are detected in frames 5 and 57 , respectively. This is due to a lack of any major difference in motion between consecutive frames. This can considerably affect the recognition performance. On the other hand, local representations are less sensitive to noise and partial occlusion. They do not need any preprocessing, such as background subtraction or tracking.

ROI representations [2], [3], [4] are obtained in a topdown fashion: a ROI is localised first in every frame. The ROI information from all the frames thus obtained constitutes the video descriptor. For human action recognition, human body parts are intuitively the vital regions of interest. These representations are powerful since they try to capture the most important human body information. For instance, in a basketball scene as shown in Fig. 1 (middle row), many body parts are detected in each frame. However, these rely heavily on accurate localisation and are dependent on the viewpoint, noise and occlusions. Moreover, they do not at all capture any contextual (scene) information. For example, when two persons are playing basketball, using only body part information is insufficient for action recognition. As can be observed in frames 43 and 57 in Fig. 1, information about the basketball is also crucial, which is a very useful example that for some action classes the interaction between human and object is crucial. As the number of action classes considered in human action recognition tasks increases, owing to the contextual information (in single or multiple person situations, with or without objects), the performance of these representations can drastically decrease.

Recently, a dense sampling approach has been proposed for human action recognition. Wang et al. [5] sampled interest points from video frames at uniform intervals in space and time and reported outperforming results of spatio-temporal interest point detectors. Further, Wang et al. [6] proposed to sample feature points on a dense grid in each frame and to track them using a state-of-the-art dense optical flow algorithm. As the feature points are dependent on tracking, they inherit the same shortcomings accrued in tracking process. For example, see frames 5, 43 and 57 in Fig. 1 (bottom row) where the second person is not detected due to a lack of sufficient motion difference between consecutive frames.

In this paper, we review and conduct extensive experiments on the three kinds of representations for large scale human 

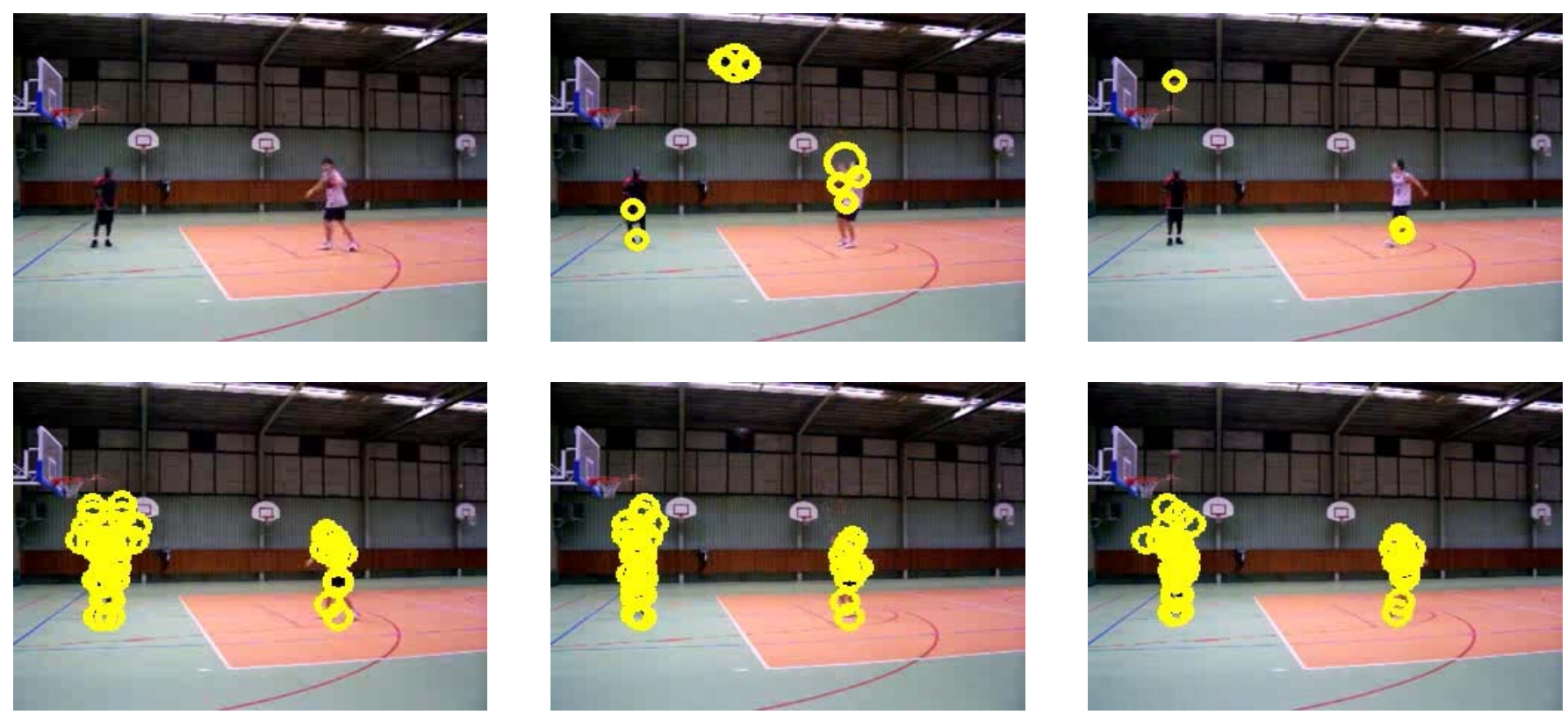

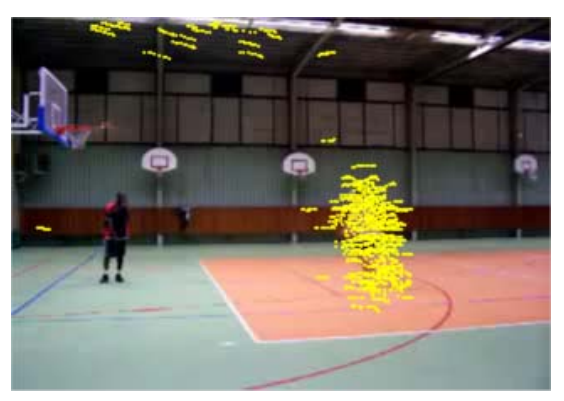

Frame \# 5

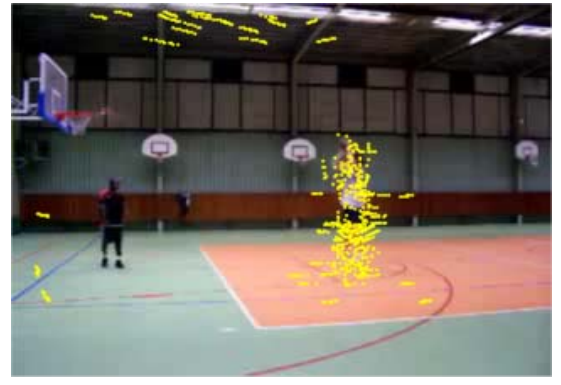

43

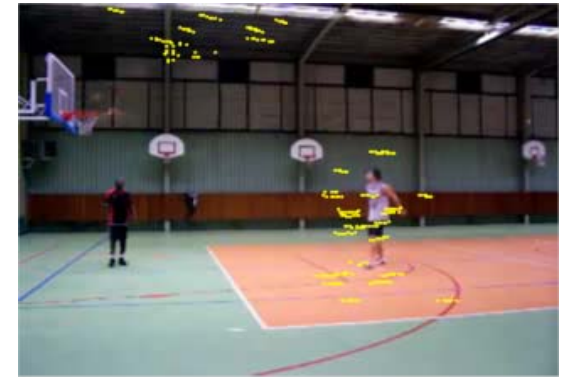

57

Fig. 1: Top: Harris 3D points require a substantial difference in motion between consecutive frames for their detection. Middle: Body part interest points do not capture the context information of the scene. Bottom: Dense trajectory interest points capture more information but still are dependent on there being a substantial difference in motion between consecutive frames.

action recognition with a particular focus on the effect of human body part representations. Although several techniques advocate the importance of human body part, they report their findings only on the KTH [7], Weizmann [8], UCF [9] and HumanEva [10] datasets, which are limited to only 6-12 action classes in fairly constrained environments. We study and present our results on the larger scale action datasets UCF50 and HMDB51, containing at least 50 different action classes each. In this regard, the following questions are investigated:

1) Do human body part representations have a substantial influence on the performance of large scale human action recognition?

2) How well do the human body part representations perform when fused with local representations?

3) How well do the human body part representations perform when fused with densely sampled representations?

We perform fusion at two levels - early (feature level) and late (classifier score) - and summarise our observations. All experiments are performed in a Bag-of-Words (BoW) framework using a Support Vector Machine (SVM) classifier.

The rest of the paper is organised as follows. Section II contains a review of local, body part (ROI), and densely sampled representations. Section III contains the details of the fusion techniques, descriptors, codebook generation, classifier and datasets used in the current study. Section IV describes the results obtained on the benchmark datasets, followed by a discussion. Finally, conclusions are drawn in Section V.

\section{Feature Representations}

In any kind of feature representation, firstly, interest points at different spatio-temporal locations and scales are detected in the given video. Then, local descriptors are computed in the spatio-temporal neighbourhood of the detected interest points. These descriptors capture shape (gradient) or motion (optical flow) information or other similar measurements meant to capture the dynamics of human actions. While the local descriptors computed in any type of representation remain the same, the detection of interest points differs due to different choices of salient content focussed on. Such interest points can be broadly classified into the following three major categories: 


\section{A. Local Interest Points}

In their seminal work, Laptev et al. [1] proposed the usage of Harris 3D corners as an extension of traditional (2D) Harris corner points for spatio-temporal analysis and action recognition. These interest points are local maxima of a function of space-time gradients. They compute a spatio-temporal secondmoment matrix at each video point in different spatio-temporal scales. This matrix essentially captures space-time gradients. The interest points are obtained as local maxima of a function of this second-moment matrix. Messing et al. [11] tracked Harris 3D interest points with a Kanade-Lucas-Tomasi (KLT) tracker to get a richer set of interest points. This yielded better performance than by Harris $3 \mathrm{D}$ points alone.

Cuboid detectors were proposed in [12]. Interest points are obtained as local maxima of the response function of temporal Gabor filters on the video. Willems et al. [13] proposed a Hessian interest point detector. This is a spatiotemporal extension of the Hessian saliency measure for blob detection in images. The detector measures the saliency with the determinant of the 3D Hessian matrix, which is computed in octaves of both spatial and temporal scales. A non-maximum suppression algorithm selects joint extrema over space, time and scales, and thus yields the interest points.

The drawback of most of these representations is that the detected points are sometimes too few in number and very much dependent on the type of salient function (gradient, Hessian, etc.) used for optimisation. Local interest points based methods can miss the global information and trigger only when spatio-temporal changes occur. In our experiments, we use the Harris 3D detectors.

\section{B. Human Body Part Interest Points}

In this representation, the body part regions are the basis for detecting interest points. Different techniques have been employed to detect the body part regions. In an early work, Song et al. [14] proposed a pose estimation based framework detecting corners in images and searching over these points for an action-specific pose model. However, this technique performs poorly in cluttered background images.

Niebles et al. [15] employed an edge detector to get an edge map of each frame and learn the relation between each body part by means of a hierarchical framework. However, the usage of an edge detector limits the efficiency of their approach, especially in cluttered or dynamic backgrounds.

Bourdev and Malik [16] proposed a two-layer classification / regression model for detecting a human body. In this context, they proposed so called "poselets", which can describe any part of a human pose including, for example, "half of a frontal face and a left shoulder" or "the legs of a person making a step in a profile view". Given a set of such 256 poselets (linear support vector machines), the input image is scanned at multiple scales. The outputs are used to vote for the location of the torso bounds or body parts such as arms, legs or face.

In Bhaskar et al. [2], three components (head, legs and arms) were detected in each video frame. The features obtained from these components were then processed by a combination of Support Vector Machines, Gaussian Mixture Models
(GMM) and Hidden Markov Models (HMM) for action recognition. They yielded a very good performance on the KTH, Weizmann and HumanEva datasets.

Felzenszwalb et al. [17], [18] proposed an object detection based framework for human body detection by parts. They model the human body as a collection of parts arranged in a deformable configuration. This deformable configuration is trained from images labelled with a bounding box around the human body without the need of detailed knowledge of the parts. The body part location is handled as a latent variable and trained through a latent support vector machine (LSVM).

Tran et al. [3] proposed another framework based on modelling the motion of human body parts. A pictorial structure model [19] was used to identify 9 human body parts in each frame of the video (head, left / right upper / lower arms, left / right upper / lower legs). The motion of each body part is then tracked and transformed into a polar histogram, modelling every human action in terms of these 9 histograms. A sparse representation framework is then employed for action recognition.

Wang and Mori [4] employed optical flow to detect motion in human body parts. They then proposed an approach that learned the parameters of a Hidden Conditional Random Field (HCRF) model in a max-margin framework for human action recognition. However, it requires accurate tracking of body parts, which is a separate, challenging task in its own right.

The drawback of all of these techniques is that they rely heavily on accurate localisation and are dependent on the viewpoint, noise and occlusions. Furthermore, they do not at all capture any contextual (scene) information. In large scale human action recognition, contextual information plays a significant role. The performance of body-part representations can drastically decrease in such scenarios. In our experiments, we use the part-based detection framework [20] to detect body part interest points (see Section III-B).

\section{Densely Sampled Interest Points}

In the dense sampling approach [5], video blocks are extracted at regular locations and scales in space and time. Length, width, time, spatial and temporal scales are the 5 dimensions that these blocks are sampled from. Spatial and temporal sampling is often done with $50 \%$ overlap.

Wang et al. further extend the dense sampling approach [5] in [21]. They first densely sample points on a uniformly spaced grid spread over the entire video in space and time. By experimentation, they found that a sampling step size of $W=5$ pixels is dense enough to give good results over several benchmark datasets. They then track these sampled points through the video. Firstly, points in homogeneous regions are removed by applying the criterion of Shi and Tomasi [22]. The optical flow field between two consecutive frames is computed on each spatial scale separately by algorithm proposed by Farneback [23]. Points of subsequent frames are concatenated to form trajectories (sets of interest points). To take care of potential drifting from the interest points' initial locations during the tracking process, tracking is performed on a fixed length $L$ number of frames at a time. In a post-processing stage, trajectories with sudden large displacements are also removed. 


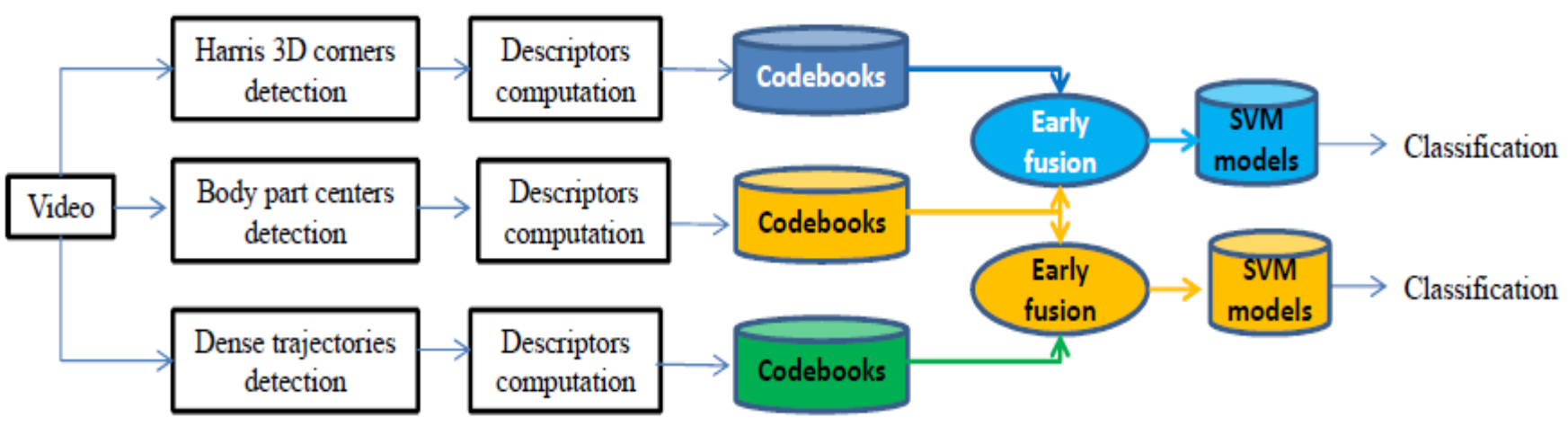

(a)

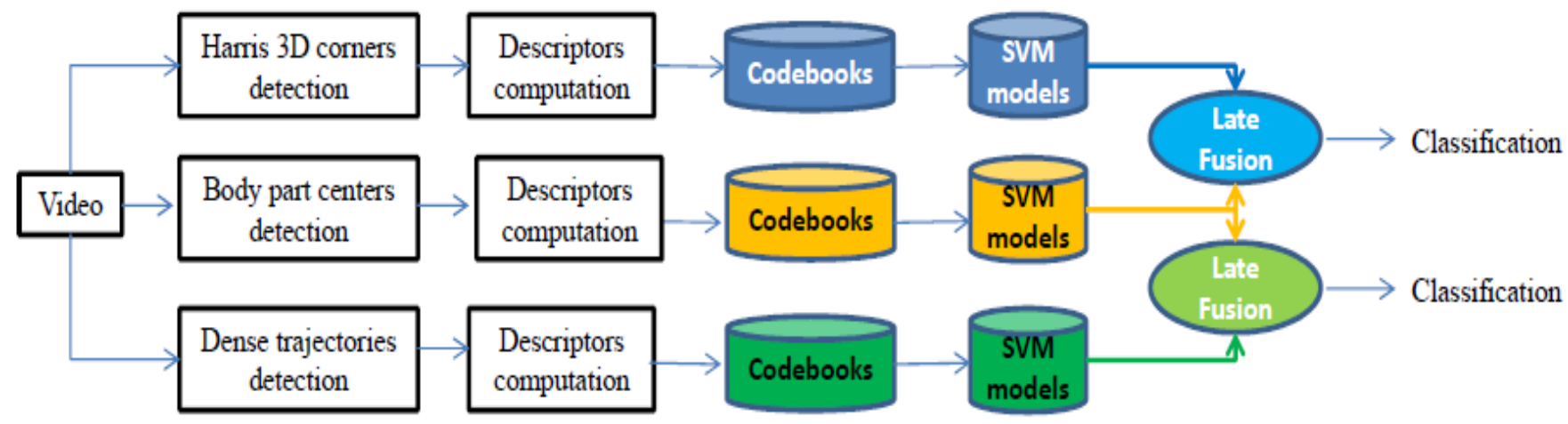

(b)

Fig. 2: BoW based frameworks are employed for each type of feature representation: Harris 3D corner points, body part interest points and densely sampled interest points. Body part interest points are fused with Harris 3D corner points or densely sampled interest points in (a) early or (b) late fusion style.

The drawback of these approaches [5],[21] is, firstly, their requirement of a huge amount of memory. This makes it inept for real-time applications. Further, their success is heavily dependent on the optical flow tracking employed, inheriting the drawbacks of optical flow tracking in general. In our experiments, we use the dense trajectory interest points.

\section{PROPOSED EXPERIMENTS}

The overall layout of our BoW based framework is shown in Fig. 2. Firstly, interest points (Harris 3D or body part or dense trajectory) are detected separately. Local descriptors are computed at the detected interest points. These descriptors are clustered into a pre-defined number of centres. The cluster centres act like visual words and thus constitute a codebook for the rest of the process. Then, visual words are used to quantise the local descriptors extracted from any given video clip into feature vectors. A video clip can be represented as a frequency histogram of the visual words, which act as features to learn a classifier. Separate codebooks are constructed for each descriptor of each feature representation

Two fusion approaches are studied to investigate the effect of body part interest points on large scale human action recognition. They are

- Early fusion - Feature histograms (of local descriptors) are concatenated before training a classifier (Fig. 2(a)). Features are first generated for each type of representation using their respective codebooks, before being concatenated, i.e. body parts with Harris 3D and body parts with dense trajectories, to yield the overall feature used to train two separate SVM framework models. We also study the multichannel approach [24] (see Section III-E).

- Late fusion - Separate classifiers are learnt for each type of local descriptor from each feature representation (Fig. 2(b)). SVM framework models are built for each feature representation separately. The decision scores obtained from each model are fused to yield the final decision of a test video's identity. To fuse two decision scores, say $d_{1}$ and $d_{2}$, both the mean and the product were experimented on.

Let us look at the details of the different components.

\section{A. Harris 3D Corner Interest Points}

These points are extracted at multiple scales based on a regular sampling of spatial and temporal scale values. They are defined in 5 dimensions $(x, y, t, \sigma, \tau)$, where $x, y$ and $t$ are spatial and temporal axes, resp., while $\sigma$ and $\tau$ are the spatial and temporal scales, respectively. We use the original implementation $^{1}$ with standard parameter settings.

\footnotetext{
${ }^{1}$ http://www.di.ens.fr/ laptev/download.html\#stip
} 


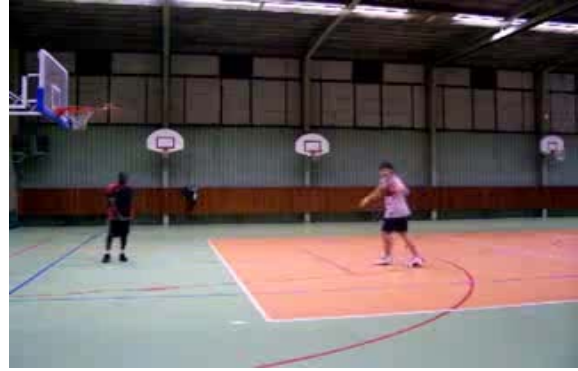

(a)

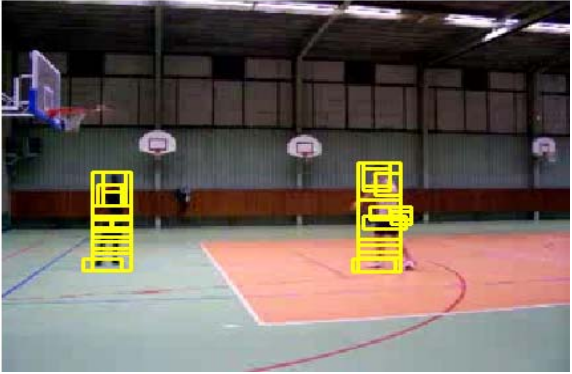

(b)

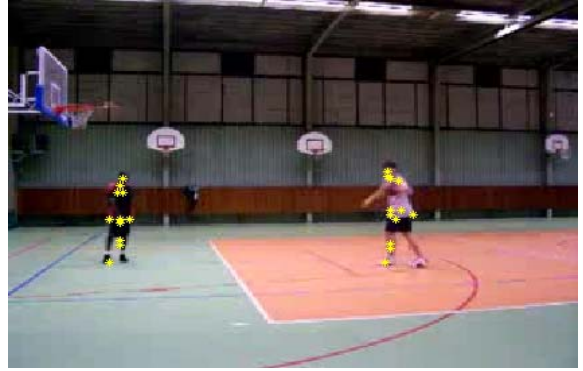

(c)

Fig. 3: Extraction of body part interest points from a video frame. (a) Original video frame. (b) shows the various candidate body parts detected using a part-based model [20]. (c) shows the centroid of the detected part regions that are considered as body part interest points in the current study.

\section{B. Body Part Interest Points}

The literature presents a number of implementations of human body detectors from still images, with the code available online. Yang and Ramanan [25] proposed a deformable part model based method for detecting a human body and estimating its pose from static images. Instead of using articulated body parts separately, they capture the orientation by a mixture of templates for each body part. The resulting flexible mixture model captures contextual co-occurrence relations between different parts, augmenting standard spring models that encode spatial relations. However, utilising the dependencies between the different parts needs the existence of either the full body or the upper body in the image. This approach would fail when this condition is not met.

Another option to detect the upper body parts is proposed by Ferrari et al. [26]. They employ a pictorial structure (PS) based pose search framework for near-frontal viewpoint images. Using a HOG based sliding window approach, candidate body regions are first detected. By using 'grab cut' segmentation, background clutter is removed and potential body regions are finalised. Using the image parsing technique of Ramanan [27], an initial pose estimate is obtained. Body part positions are then detected using an integrated spatiotemporal model refining the initial pose estimate. Again, the drawback of this approach is that no body parts below the torso are detected as well as the need for the existence of the upper body parts.

In action recognition datasets, such as HMDB51 and UCF50, the full body is visible in classes such as 'walk' or 'throw'. However, in other classes such as 'smile' or 'kiss', the face is the only part that is visible. For the sake of generality, we employ a different, in our opinion better, approach than both of the above techniques, which has been proposed by Girshick et al. [20]. Their method can be utilised to detect the person, fully or partially visible. They organise the human body as (1) Terminals, (2) Non-terminals and (3) Subtypes (which can be derived from the non-terminals). The terminals are 6 parts, beginning with the head, shoulders, torso, and finally the legs. They also added an extra 'body part' for handling the occlusion (missing parts) resulting in a total of 7 parts. For improved efficiency, these parts are flipped, resulting in 14 parts. Several rules constraining deformation and structure of body parts were proposed so as to increase the flexibility of the learnt model when facing large variability in the positions of the human parts. Unlike other approaches, this detector works reasonably well in non-frontal poses and partially occluded body part situations. Hence, we choose this implementation in our approach.

Using the original implementation ${ }^{2}$, candidate body parts are extracted from every third frame of the given video. Reddy and Shah [28] reported in their experiments that features extracted from every third frame will not affect the overall performance significantly. As shown in Fig. 3, the body parts are detected first by applying the part-based model for human bodies. The centroid of each detected body part region is considered as an interest point in that particular frame. These interest points accumulated over an entire video yield the video's body part interest points. There is no ground truth for human body parts for the UCF50 and HMDB51datasets.

\section{Local Descriptors}

Local descriptors are computed at the detected interest points to capture characteristics such as appearance and motion. Following the extensive evaluation of several detectors in [5], we computed histograms of gradient orientations (HOG) and histograms of optic flow (HOF) descriptors. While the former captures the local motion and appearance, the latter captures the temporal changes. For the computation of $\mathrm{HOG} / \mathrm{HOF}$ descriptors, the descriptor volume is defined by $\Delta_{x}(\sigma)=\Delta_{y}(\sigma)=18 \sigma, \Delta_{t}=8 t$. Each volume is subdivided into a $n_{x} \times n_{y} \times n_{t}$ grid of cells. For each cell, 4-bin HOG and 5-bin HOF descriptros are computed. Normalised histograms are concatenated to yield the final $\mathrm{HOG} / \mathrm{HOF}$ descriptor vectors. In our experiments, we use the parameters $n_{x}, n_{y}=3, n_{t}=2$ as in the original implementation ${ }^{1}$.

In relation to dense trajectory based representations, two more additional descriptors are computed. They are Motion Boundary Hisotgrams (MBH) and Trajectory shape. The dense trajectories code available on-line ${ }^{3}$ [6] is used in our experiments. Motion Bound Histograms are descriptors based on motion boundaries. Initially developed in the context of human detection by Dalal et al. [29], these descriptors are computed by separate derivatives for the horizontal and vertical

\footnotetext{
${ }^{2}$ http://people.cs.uchicago.edu/ rbg/latent/

${ }^{3} \mathrm{http}: / /$ lear.inrialpes.fr/software
} 
components of the optical flow. As MBH captures the gradient of the optical flow, constant camera motion is removed and information about changes in the flow field (i.e. motion boundaries) are retained. An 8-bin histogram is obtained along each component of $x$ and $y$. Both histogram vectors are normalised separately with their $L_{2}$ norm, each resulting in a vector of length 96. The Trajectory shape descriptor encodes local motion patterns. For a trajectory of given length $L$ (= number of frames), the trajectory shape is computed as a sequence of displacement vectors of points $(x, y)$ of subsequent frames. The resulting vector is normalised by the sum of the displacement vector magnitudes. We take $L=15$ frames (default settings) and obtain a 30-dimensional descriptor as in the original implementation.

\section{Codebooks and Encoding}

We built separate codebooks for each descriptor in each feature representation. In our experiments, codebooks are constructed with k-means clustering. We set the number of visual words $V$ to 4000, which was shown to give good results [5]. Experimental settings set in [30] were followed. We cluster a subset of 100,000 randomly selected local descriptors to find the visual words. We also initialise the k-means clustering 8 times and keep the result with the lowest error.

A major step in constructing a BoW representation is encoding a feature with the codebook. The Hard Assisgnment (HA) scheme based on the nearest Euclidean distance is the foremost technique in this regard. In this scheme, local descriptors are assigned to their closest vocabulary words. Recently, a few other encoding methods such as Soft Assignment encoding [31], sparse encoding [32], locality-constrained linear encoding [33] and Fisher kernel encoding [34] have been proposed for general object recognition. Their performance on human action recognition has not been explored yet. Xingxing et al. [35] did some ground work in this direction evaluating and comparing encoding, pooling and normalisation methods in the context of video based action recognition. To maintain consistency, we used only the HA scheme in all our experiments.

\section{E. Classification}

We use a non-linear SVM with an RBF- $\chi^{2}$ kernel classifier as in [5]. We compute the $\chi^{2}$ distance for each pair of training feature vectors and obtain the kernel matrix. We normalise this kernel matrix using the average $\chi^{2}$ distance value $(A)$ of the training samples within themselves. We then map this Kernel matrix using the exponential function $e^{-x}$, i.e.

$$
K\left(H_{i}, H_{j}\right)=\exp \left(-\sum_{c} \frac{1}{2 A^{c}} \frac{\left(H_{i}-H_{j}\right)^{2}}{H_{i}+H_{j}}\right)
$$

where $c$ is the number of channels (of descriptors). $H_{i}=h_{\text {in }}$ and $H_{j}=h_{j n}$ are the frequency histograms of word occurrences for the $c^{\text {th }}$ channel descriptor.

This transformed kernel matrix is then passed on to an SVM with Radial Basis Kernel [36]. We have fixed the hyper parameter $C=100$ in all our experiments. For multi-class classification, we apply the one-versus-all approach and select the class with the highest score.

\section{F. Datasets}

We carry out our experiments on the benchmark datasets UCF50 [28] and HMDB51 [37]. The choice of datasest is motivated by wanting to focus on datasets having the largest number of classes till date. UCF50 has 50 action classes. $^{4}$ This dataset consists of 6680 realistic videos collected from YouTube. As specified in [28], we perform a 25-fold groupwise cross-validation classification experiment on this dataset. HMDB51 contains 51 actions categories. ${ }^{5}$ Digitised movies, public databases such as the Prelinger archive, videos from YouTube and Google videos were used to create this dataset. For evaluation purposes, three distinct training and testing splits are specified in the dataset. These splits were built to ensure that clips from the same video were not used for both training and testing. For each action category in a split, 70 training and 30 testing clips indices were fixed so that they fulfil the 70/30 balance for each meta tag.

\section{EXPERIMENTS AND DISCUSSIONS}

We now present our results and discuss the questions we raised in the introduction. Tables I and III present the summary of the results in comparison with current state-ofthe-art methods in the literature.

\section{A. Can different human body part representations have sub-} stantial influence on large scale human action recognition?

Body part based recognition rates are shown in Table I. These are $61.7 \%$ and $24.2 \%$ on UCF50 and HMDB51, respectively. The performance when using Harris 3D corner points was $69.8 \%$ and $31.3 \%$, respectively. They performed $6-7 \%$ (absolute) better than the body part based frameworks. The difference can be attributed to the reason we mentioned earlier, i.e. body part interest points do not capture any context information. Many of the classes, in UCF50 or HMDB51, are very similar. For instance, in the HMDB51 dataset 'Shoot ball', 'Shoot bow', 'Shoot gun'; or 'Climb', 'Climb Stairs' and so on. Similarly in the UCF50 dataset, 'Horse Race', 'Horse Riding'; 'Jump rope', 'Jumping Jack'; 'Rock climbing indoor', 'Rope climbing' and so on. In such situations, context (scene, object) information is very crucial. As this contextual information cannot be captured by body parts, a lower performance than in the Harris 3D based framework is not surprising.

From the results obtained on datasets containing at least 50 classes, it can be noted that body part based interest points are worthwhile, especially, when these videos are collected from uncontrolled settings like YouTube, Google, etc.

\section{B. How well do the human body part representations perform when fused with local representations?}

The results for early (feature level) and late (classifier score level) fusion are shown in Table I. The best performance scores obtained on UCF50 and HMDB51 are $72.2 \%$ and $35.3 \%$, respectively. In both cases, there has been an improvement over the Harris 3D corner and body part interest point based frameworks by as much as 3-4\% (absolute). This also supports the hypothesis that local descriptor and body part frameworks complement each other.

\footnotetext{
${ }^{4}$ Dataset available at http://crcv.ucf.edu/data/UCF50.php

${ }^{5}$ Dataset available at http://serre-lab.clps.brown.edu/resources/HMDB/
} 
TABLE I: Harris 3D corner points and body part based recognition rates.

\begin{tabular}{|c|c|c|c|}
\hline \multicolumn{2}{|c|}{ Approach and Descriptor } & UCF50 & HMDB51 \\
\hline \multirow{2}{*}{ Body part based } & HOG (A) & $46.8 \%$ & $16.3 \%$ \\
\hline & $\mathrm{HOF}(\mathrm{B})$ & $49.3 \%$ & $18.1 \%$ \\
\hline Combined & multichannel & $61.7 \%$ & $24.2 \%$ \\
\hline \multirow[t]{2}{*}{ Harris 3D based } & HOG (C) & $57.0 \%$ & $21.2 \%$ \\
\hline & $\mathrm{HOF}$ (D) & $57.0 \%$ & $23.4 \%$ \\
\hline Combined & Multichannel & $69.8 \%$ & $31.4 \%$ \\
\hline \multirow{2}{*}{$\begin{array}{l}\text { Early fusion } \\
(\mathrm{A}+\mathrm{B}+\mathrm{C}+\mathrm{D})\end{array}$} & Multichannel & $71.9 \%$ & $35.1 \%$ \\
\hline & Concatenation & $71.2 \%$ & $35.3 \%$ \\
\hline \multirow{2}{*}{$\begin{array}{l}\text { Late Fusion } \\
(\mathrm{A}+\mathrm{B}+\mathrm{C}+\mathrm{D})\end{array}$} & Product & $72.2 \%$ & $34.1 \%$ \\
\hline & Mean & $70.1 \%$ & $30.8 \%$ \\
\hline \multicolumn{2}{|c|}{ Kuehne et al. 2011 [37] } & $47.9 \%$ & $22.8 \%$ \\
\hline \multicolumn{2}{|c|}{ Sadanand and Corso 2012 [38] } & $57.9 \%$ & $26.9 \%$ \\
\hline \multicolumn{2}{|c|}{ Kliper-Gross et al. 2012 [39] } & $72.6 \%$ & $29.2 \%$ \\
\hline \multicolumn{2}{|c|}{ Solmaz et al. 2012 [40] } & $73.7 \%$ & $29.2 \%$ \\
\hline \multicolumn{2}{|c|}{ Reddy and Shah [28] } & $68.5 \%$ & - \\
\hline \multicolumn{2}{|c|}{ Xingxing et al. 2012 [35] } & - & $31.84 \%$ \\
\hline
\end{tabular}

\section{How well do the human body part representations perform} when fused with densely sampled representations?

Motion Boundary Histograms (MBH) descriptors were computed along $x$ and $y$ directions separately. Codebooks and SVMs were further built on them. The performance scores of these descriptors on UCF50 and HMDB51 are shown in Table II. Using the multi-channel approach, the overall $\mathrm{MBH}$ shape performance was computed. The resulting performance on UCF50 and HMDB51 were $83.2 \%$ and $43.5 \%$, respectively.

TABLE II: Performance of $\mathrm{MBH}$ along $x$ and $y$ directions for dense trajectories on UCF50 and HMDB51 datasets, plus their combination using the multi-channel approach.

\begin{tabular}{|c|c|c|c|}
\hline Dataset & $x$ direction & $y$ direction & Combined \\
\hline \hline UCF50 & $78.2 \%$ & $77.2 \%$ & $83.2 \%$ \\
\hline HMDB51 & $32.1 \%$ & $37.9 \%$ & $43.5 \%$ \\
\hline
\end{tabular}

Recognition rates for dense trajectory representations are shown in Table III. They achieved $84.5 \%$ and $45.5 \%$ on UCF50 and HMDB51, respectively. Upon fusing with the body part based information, the best recognition rates obtained were $84.9 \%$ and $47.5 \%$, respectively. An increase of 0.4-2\% (absolute) has been observed.

\section{Computational Considerations}

We now give an idea of the time taken and the number of interest points detected for each type of feature representation. Our experiments were carried out on a server with 16-core Xeon processor $(2.4 \mathrm{GHz})$ and 64GB RAM. For a typical video containing 107 frames (duration of 3s) and frame size $320 \times$ 240 pixels, the statistics are shown in Table IV. Only the body parts detection used the multi-core features of the server. The other two representations ran on single core instances only.
TABLE III: Dense trajectory based recognition rates

\begin{tabular}{|c|c|c|c|}
\hline \multicolumn{2}{|c|}{ Approach and Descriptor } & UCF50 & HMDB51 \\
\hline \hline Trajectory based & HOG (E) & $67.6 \%$ & $26.8 \%$ \\
& HOF(F) & $69.5 \%$ & $23.4 \%$ \\
& Traj. Shape (G) & $68.5 \%$ & $31.6 \%$ \\
& MBH (H) & $83.2 \%$ & $43.5 \%$ \\
Combined & Multichannel & $84.5 \%$ & $45.5 \%$ \\
\hline \hline Early fusion & Multichannel & $83.5 \%$ & $45.7 \%$ \\
(E+F+G+H+C+D) & Concatenation & $78.9 \%$ & $32.9 \%$ \\
\hline Late Fusion & Product & $\mathbf{8 4 . 9 \%}$ & $\mathbf{4 7 . 5 \%}$ \\
(E+F+G+H+C+D) & Mean & $84.3 \%$ & $45.2 \%$ \\
\hline \hline \multicolumn{2}{|c|}{ Jiang et al. $2012[41]$} & - & $40.7 \%$ \\
\hline \multicolumn{2}{|c|}{ Wang et al. $2013[21]$} & $84.5 \%$ & $46.6 \%$ \\
\hline \multicolumn{2}{|c|}{ Reddy and Shah 2012 [28] } & $76.9 \%$ & - \\
\hline
\end{tabular}

TABLE IV: Computational considerations for a typical video for different kinds of feature representations.

\begin{tabular}{|c|c|c|}
\hline Feature type & Execution time & Interest points \\
\hline Harris 3D & $43.3 \mathrm{~s}$ & 1,940 \\
\hline Dense trajectories & $38.6 \mathrm{~s}$ & 20,749 \\
\hline Body part & $214.3 \mathrm{~s}$ & 1,467 \\
\hline
\end{tabular}

\section{E. Comparison with Other Techniques}

The objective of this study was to apply different types of feature representations and to investigate the effect of body part interest points in large scale human action recognition. An attempt to combine with STIP (Harris 3D corner points) and densely sampled and trajectory based frameworks was also performed. We observe that the best fusion results are better than state-of-the-art methods, as shown in Table III. Although the information from the body parts is not producing high performance on its own, it does play a role in large scale human action recognition, when it is fused with Harris 3D corner interest points or dense trajectory based techniques.

\section{CONCLUSiOnS}

In this paper, three different kinds of feature representations have been studied and experimented on for the purpose of human behaviour analysis in large scale action recognition. The role of human body part interest points was another point in focus. A Harris 3D corner (local representation) based BoW framework performed $7-8 \%$ (absolute) better than a human body part based framework. Nevertheless, body part interest point information was found to be useful. Upon fusion with the Harris 3D based framework, overall performance improved by 3-4\% (absolute). Densely sampled and trajectory based interest points fared the best among all three kinds of feature representations employed. Fusion of body part information yielded an improvement of $0.4-2 \%$ (absolute). The information from body parts does play a role in large scale action recognition, when it is fused with Harris 3D corner points or dense trajectory based techniques as the information complements each other.

\section{REFERENCES}

[1] I. Laptev and T. Lindeberg, "Space-Time Interest Points," in International Conference on Computer Vision (ICCV), Oct 2003, pp. 432-439. 
[2] B. Chakraborty, A. Bagdanov, J. Gonzalez, and X. Roca, "Human Action Recognition Using an Ensemble of Body-Part Detectors," Expert Systems, 2011.

[3] K. Tran, I. Kakadiaris, and S. Shah, "Modeling motion of body parts for action recognition," in British Machine Vision Conference (BMVC), 2011, pp. 64.1-64.12.

[4] Y. Wang and G. Mori, "Hidden part models for human action recognition: Probabilistic versus max margin," IEEE Transactions on Pattern Analysis and Machine Intelligence, vol. 33, no. 7, pp. 1310 -1323, July 2011.

[5] H. Wang, M. M. Ullah, A. Kläser, I. Laptev, and C. Schmid, "Evaluation of local spatio-temporal features for action recognition," in British Machine Vision Conference (BMVC), Sep 2009, p. 127.

[6] H. Wang, A. Klaser, C. Schmid, and C.-L. Liu, "Action recognition by dense trajectories," in IEEE Conference on Computer Vision and Pattern Recognition (CVPR), June 2011, pp. 3169-3176.

[7] C. Schuldt, I. Laptev, and B. Caputo, "Recognizing human actions: a local svm approach," in International Conference on Pattern Recognition (ICPR), vol. 3, 2004, pp. 32-36.

[8] M. Blank, L. Gorelick, E. Shechtman, M. Irani, and R. Basri, "Actions as Space-Time Shapes," in International Conference on Computer Vision (ICCV), 2005, pp. 1395-1402.

[9] M. Rodriguez, J. Ahmed, and M. Shah, "Action mach a spatio-temporal maximum average correlation height filter for action recognition," in IEEE Conference on Computer Vision and Pattern Recognition (CVPR), June 2008, pp. 1-8.

[10] L. Sigal, A. O. Balan, and M. J. Black, "Humaneva: Synchronized video and motion capture dataset and baseline algorithm for evaluation of articulated human motion," International Journal on Computer Vision, vol. 87, no. 1-2, pp. 4-27, Mar 2010.

[11] R. Messing, C. Pal, and H. Kautz, "Activity recognition using the velocity histories of tracked keypoints," in International Conference on Computer Vision (ICCV), 2009.

[12] P. Dollár, V. Rabaud, G. Cottrell, and S. Belongie, "Behaviour recognition via sparse spatio-temporal features," in IEEE International Workshop on Visual Surveillance and Performance Evaluation of Tracking and Surveillance. IEEE, Oct. 2005.

[13] G. Willems, T. Tuytelaars, and L. Gool, "An efficient dense and scale-invariant spatio-temporal interest point detector," in European Conference on Computer Vision, ser. ECCV, 2008, pp. 650-663.

[14] Y. Song, L. Goncalves, and P. Perona, "Unsupervised learning of human motion," IEEE Transactions in Pattern Analysis and Machine Intelligence, 2003.

[15] J. Niebles and L. Fei-Fei, "A hierarchical model of shape and appearance for human action classification." in IEEE Conference on Computer Vision and Pattern Recognition (CVPR), 2007.

[16] L. Bourdev and J. Malik, "Poselets: Body Part Detectors Trained Using 3D Human Pose Annotations," in International Conference on Computer Vision (ICCV), 2009.

[17] P. Felzenszwalb, D. McAllester, and D. Ramanan, "A discriminatively trained, multiscale, deformable part model," in IEEE Conference on Computer Vision and Pattern Recognition (CVPR), 2008.

[18] P. F. Felzenszwalb, R. B. Girshick, D. McAllester, and D. Ramanan, "Object Detection with Discriminatively Trained Part Based Models," IEEE Transactions on Pattern Analysis and Machine Intelligence, vol. 32, no. 9, pp. 1627-1645, 2010.

[19] P. F. Felzenszwalb and D. P. Huttenlocher, "Pictorial structures for object recognition," International Journal on Computer Vision, vol. 61, no. 1, pp. 55-79, Jan. 2005.

[20] R. B. Girshick, P. Felzenszwalb, and D. Mcallester, "Object detection with grammar models," in Advances in Neural Information Processing Systems 24, 2011, pp. 442-450.

[21] H. Wang, A. Klser, C. Schmid, and C.-L. Liu, "Dense trajectories and motion boundary descriptors for action recognition," International Journal of Computer Vision, vol. 103, no. 1, pp. 60-79, 2013.

[22] J. Shi and C. Tomasi, "Good features to track," in IEEE Computer Vision and Pattern Recognition (CVPR), 1994, pp. 593-600.

[23] G. Farnebäck, "Two-frame motion estimation based on polynomial ex- pansion," in Scandinavian Conference on Image Analysis, ser. SCIA'03. Springer-Verlag, 2003, pp. 363-370.

[24] J. Zhang, M. Marszalek, S. Lazebnik, and C. Schmid, "Local features and kernels for classification of texture and object categories: A comprehensive study," International Journal of Computer Vision, vol. 73, no. 2, pp. 213-238, Jun. 2007.

[25] Y. Yang and D. Ramanan, "Articulated pose estimation with flexible mixtures-of-parts," in IEEE Conference on Computer Vision and Pattern Recognition (CVPR 2011), 2011, pp. 1385-1392.

[26] V. Ferrari, M. Marin-Jimenez, and A. Zisserman, "Progressive search space reduction for human pose estimation," in IEEE Conference on Computer Vision and Pattern Recognition (CVPR), Jun 2008.

[27] D. Ramanan, "Learning to parse images of articulated bodies," in Advances in Neural Information Processing Systems 19, B. Schölkopf, J. Platt, and T. Hoffman, Eds. MIT Press, 2007, pp. 1129-1136.

[28] K. K. Reddy and M. Shah, "Recognizing 50 Human Action Categories of Web Videos," Machine Vision and Applications, vol. 24, pp. 971-981, Sept 2012.

[29] N. Dalal, B. Triggs, and C. Schmid, "Human detection using oriented histograms of flow and appearance," in European conference on Computer Vision, ser. ECCV. Springer-Verlag, 2006, pp. 428-441.

[30] M. Marszalek, I. Laptev, and C. Schmid, "Actions in context," IEEE Conference on Computer Vision and Pattern Recognition (CVPR), 2009.

[31] L. Liu, L. Wang, and X. Liu, "In defense of soft-assignment coding," in International Conference on Computer Vision (ICCV), 2011, pp. 24862493.

[32] J. Yang, K. Yu, Y. Gong, and T. Huang, "Linear spatial pyramid matching using sparse coding for image classification," in IEEE Conference on Computer Vision and Pattern Recognition (CVPR), 2009, pp. 17941801.

[33] J. Wang, J. Yang, K. Yu, F. Lv, T. Huang, and Y. Gong, "Localityconstrained linear coding for image classification," in IEEE Conference on Computer Vision and Pattern Recognition (CVPR), 2010, pp. 33603367.

[34] F. Perronnin, J. Sánchez, and T. Mensink, "Improving the fisher kernel for large-scale image classification," in European conference on Computer vision (ECCV), ser. ECCV'10. Springer-Verlag, 2010, pp. 143-156.

[35] X. Wang, L. Wang, and Y. Qiao, "A comparative study of encoding, pooling and normalization methods for action recognition," in Asian Conference on Computer Vision, ser. ACCV, 2012.

[36] C.-C. Chang and C.-J. Lin, "LIBSVM: A library for support vector machines," ACM Transactions on Intelligent Systems and Technology, vol. 2, pp. 27:1-27:27, 2011, software available at http://www.csie.ntu.edu.tw/ cjlin/libsvm.

[37] H. Kuehne, H. Jhuang, E. Garrote, T. Poggio, and T. Serre, "HMDB: A large video database for human motion recognition," in International Conference on Computer Vision (ICCV), nov 2011, pp. 2556-2563.

[38] S. Sadanand and J. Corso, "Action Bank: A High-Level Representation of Activity in Video," in IEEE Conference on Computer Vision and Pattern Recognition (CVPR 2012), 2012, pp. 1234-1241.

[39] T. H. Orit Kliper-Gross, Yaron Gurovich and L. Wolf, "Motion Interchange Patterns for Action Recognition in Unconstrained Videos," in European Conference on Computer Vision (ECCV 2012), Oct 2012, pp. 256-269.

[40] B. Solmaz, S. M. Assari, and M. Shah, "Classifying Web Videos using a Global Video Descriptor," Machine Vision and Applications, vol. 24, pp. 1473-1485, 2012.

[41] Y.-G. Jiang, Q. Dai, X. Xue, W. Liu, and C.-W. Ngo, "Trajectory-based modeling of human actions with motion reference points," in European Conference on Computer Vision (ECCV 2012), 2012, pp. 425-438. 